\title{
Monoclonal Antibody as an Emerging Therapies for
}

\section{Ischemic Stroke}

Demi Woods ${ }^{1}$, Qian Jiang ${ }^{1}$, Xiang-Ping Chu ${ }^{1,2}$

${ }^{1}$ Department of Biomedical Sciences, School of Medicine, University of Missouri-Kansas City, Kansas City, MO 64108, USA; demiwoods@umkc.edu (D.W.); jiangqi@umkc.edu (Q.J.); chux@umkc.edu (X.-P.C.)

${ }^{2}$ Neuroscience Laboratory for Translational Medicine, School of Mental Health, Qiqihar Medical University, Qiqihar, Heilongjiang 161006, China; chux@umkc.edu (X.-P.C.)

Correspondence and requests for materials should be addressed to

chux@umkc.edu; Tel: +1- 816-235-2248

Xiang-Ping Chu, MD, PhD

Associate Professor

Department of Biomedical Sciences

School of Medicine

University of Missouri-Kansas City

Kansas City, MO 64108, USA

Email: chux@umkc.edu

Phone: +1-816-235-2248

Fax: +1-816-235-6517 


\begin{abstract}
Acute ischemic strokes are the third leading cause of death and the leading cause of neurological disability worldwide. The oxygen and glucose deprivation associated with ischemic strokes not only leads to neuronal cell death, but also increases the inflammatory response and decreases functional output of the brain. The only intervention approved by US Federal Drug and Food Administration for treatment of ischemic strokes is tissue plasminogen activator (tPA), however, such treatment can only be given within 4.5 hours of the onset of stroke-like symptoms. This narrow time-range limits its application, and it also might induce detrimental rather than beneficial effects to stroke patients by treatment of the tPA. In order to reduce the infarct volume of an acute ischemic stroke while increasing the time period for treatment, emerging therapies reveal great potential by targeting inflammation, growth factors, ion channels, and neurotransmitter receptors with monoclonal antibody (MAB). With successfully application in the treatment of cancer patient by MAB, in this review, we will focus on recent advances on stroke therapy by using MAB on the treatment of stroke by targeting inflammation, growth factors, ion channels, and neurotransmitter receptors. Therefore, developing specific MAB targeting the signaling pathway of stroke will contribute to stroke therapy.
\end{abstract}

Key words: stroke; antibody therapy; monoclonal antibody; inflammation; acid-sensing ion channel; receptor; growth factors 


\section{Introduction}

Acute ischemic strokes are defined as a rapid decrease in blood flow to the brain immediately depriving the brain from oxygen and glucose. The lack in blood supply leads to neuronal and glia cell death and subsequent loss of function in the brain. As the third leading cause of death and the leading cause of neurological disability in the world, novel and effective therapies are critical and warranted [1].

Currently, the intervention for acute ischemic stroke treatment is intra-arterial or intravenous tissue plasminogen activator (tPA) which acts to dissolve the clot and improve blood flow and was approved by US Federal Drug and Food Administration (FDA) for acute stroke therapy. However, tPA must be given less than 4.5 hours from the onset of symptoms limiting the efficacy of the drug [2]. Aside from the narrow therapeutic window of tPA, over $50 \%$ of patients that receive tPA acutely post-stroke have significant long-term disability [3]. Due to a narrow therapeutic window and severe side effects of tPA, new treatments are needed to be accessible to a majority of patients while improving stroke outcome.

One of the major contributing factors worsening the clinical outcome of stroke patients is inflammation [3]. Pro-inflammatory cytokines contribute to brain cell death by activating a variety of cells: neurons, astrocytes, microglia, and endothelial cells. This activation leads to neuronal and glial cell death and contributes to the progression of brain injury following an ischemic stroke [2-4]. After ischemic stroke, the pro-inflammatory cytokines and antiinflammatory cytokines are activated. For example, tumor necrosis factor- $\alpha$ (TNF- $\alpha)$, interleukin-1, 8 (IL-1, IL-8) and monocyte chemoattraction protein-1 (MCP-1) are proinflammatory stroke cytokines that contribute to neuronal cell death, while interleukin-6, 10, 20 (IL-6, IL-10, IL-20), transforming growth factor- $\beta$ (TGF- $\beta$ ), and interferon-inducible protein-10 
(IP-10) are anti-inflammatory and neuroprotective [5-7]. Recent studies have shown that monoclonal antibody (MAB) reveals protection against the acute ischemic stroke [4]. MAB can be administered to block the deleterious pathways associated with inflammation by inhibiting pro-inflammatory cytokines or their receptors as well as inhibiting ion channels, which contributes to neuronal cell injury after ischemic stroke [4]. Generating specific antibodies that target the inflammatory system, ion channels or cell death pathway show a promising therapy toward managing the outcome of a stroke [4].

Emerging therapies based on neuronal regeneration also provide benefits to patients that are past the 4.5-hour time window with tPA treatment. Repair therapies can be accessible to a large patient population because they have a time window of days to weeks [3]. MAB promotes neural repair by binding to receptors or surface markers to block or neutralize inhibitors of neuronal cell growth. Three major inhibitors of neuronal cell growth are myelin-associated glycoprotein (MAG), oligo-myelin glycoprotein, and Nogo-A. Following an acute ischemic stroke, MAG, oligo-myelin glycoprotein, and Nogo-A are upregulated [8]. MAB can be used to inhibit these inhibitors and will promote neuronal repair and axonal growth [9].

Most antibodies evaluated for stroke treatment prove effective in reducing infarct volume and improving neuronal performance in animal models of middle cerebral artery occlusion (MCAO). Here, we reviewed the current MAB therapies and its specific targets on inflammation, growth factor, ion channel and receptor, which contribute to enhance neuroplasticity and improve the of outcome animals suffered from stroke. We also discussed the $\mathrm{MAB}$ in the clinical trials for stroke patients.

\section{Inflammation after Stroke}




\subsection{Inflammation Defined}

Inflammation following damage to any tissue begins as an acute process. Inflammatory molecules are upregulated in such conditions and are responsible for the local and systemic effects induced by inflammation. The main components responsible are cytokines such as ILs and TNF- $\alpha$, chemokines such as MCP-1, and various cellular adhesion molecules [10]. Inflammation has detrimental and deleterious effects on neurons. Following an ischemic stroke, elevations in various inflammatory markers provide evidence that inflammation of brain parenchyma following a stroke is neurotoxic and thus, contributes to the downfall of patients post-stroke.

\subsection{Inflammatory Pathways after MCAO}

Immunotherapy blocks specific deleterious pathways that are the direct outcome of an inflammatory response following a stroke. The results of inflammation as well as the cytokines produced post stroke are the main contributors in the damage that ensues after a stroke [11]. Not only would inhibit inflammatory pathways and prevent lifelong damage, but it would increase the timespan for which reperfusion therapy can be given to treat an acute ischemic stroke. For instance, in acute ischemic stroke, reperfusion must be given within 4.5 hours from the initial onset of symptoms. If reperfusion is administered any later than 4.5 hours, the adverse side effects as a result of the accumulation of reactive oxygen species will outweigh the initial benefit provided by the therapy. Whereas, inhibition of inflammation following an acute ischemic stroke will not only decrease the infarct volume and improve clinical outcomes, but also drugs used to suppress inflammation may also widen the therapeutic window for tPA administration beyond 
4.5 hours. Clinical trials utilizing anti-inflammatory agents in acute ischemic strokes have only been successful in animal models and have subsequently failed in human suffered from stroke $[10]$.

In order to reduce inflammation, antibodies must be administered against specific targets. Antibodies can be administered to bind to receptors and inhibit the signaling cascades. Inhibition of toll-like receptor 4 (TLR4) via MAB in MCAO mice proved beneficial in decreasing inflammation. Total infarct volume and brain swelling 48 hours after an MCAO stroke decreased in mice treated with MAB against TLR4 in comparison to MCAO mice that did not receive MAB [12].

MAB against leukocyte adhesion molecules was also evaluated. Specifically, alpha-4 integrin antibody was administered against alpha-4 integrin, a specific leukocyte adhesion molecule [13]. In MCAO mice, MAB administered against alpha-4 integrin reduced stroke volume and overall brain infarction volume, however, the study was not successful in human trials. Natalizumab is an antibody formed against leukocyte adhesion molecules, specifically alpha-4 integrin. When natalizumab, widely used in the treatment of multiple sclerosis and Crohn's disease approved by FDA, was used in patients with stroke. It did not show a significant reduction of infarct growth which is in contrast to the animal study [13].

The difference between outcomes of animal models and humans suffered from ischemic stroke might be explained by humans and mice having different post-ischemic inflammatory responses. Potentially, there might be differences in alpha-4 integrin expression between species [13]. Another possibility for the lack of reduction of infarct volume could be attributed to the dose administered. Initially, the study administered $300 \mathrm{mg}$ of natalizumab based on the dosage that is approved as a treatment of Multiple Sclerosis [13]. The study suggests doses of 
natalizumab ranging from 450-600 mg might have better clinical outcomes in regards to reduction of infarct volume compared to the original trial of $300 \mathrm{mg}$ [13]. This should be evaluated in further studies.

\section{Neuronal Repair after Stroke}

Neuronal repair is important for restoring the structure and function of neurons in central nervous system (CNS) following an injury. Without neuronal repair and regeneration, stroke patients would never have the opportunity to recover. Therapies based on neuronal repair have a much greater therapeutic window than the administration of tPA. Therapies are measured in days to weeks and have the potential of benefiting a variety of stroke patients, especially those that fall outside of the narrow therapeutic window established by tPA $[11,14]$. In particular, studies evaluated the neuronal repair process after an acute ischemic stroke. Following an acute ischemic stroke, neuronal repair occurs spontaneously and can occur for many years afterwards. Thankfully, animal studies have provided further insight into neuronal repair following an MCAO ischemic stroke and have led researchers to conclude that the majority of therapies used to promote neuronal repair focus on a single intervention and are thus termed monotherapies [14].

\subsection{Activation of Growth Factors for Neuronal Regeneration}

Growth factors are necessary for CNS development and are vital in neuronal repair. Growth factors stimulate angiogenesis, cell proliferation, cell differentiation, cell migration, cell survival, and cellular synaptic plasticity $[15,16]$. Without the activation of growth factors following an acute ischemic stroke or any CNS injury, neurons would not be able to regenerate. 
A preclinical stroke study suggests that the administration of exogenous growth factors 24 hours prior to the induction of an MCAO in an animal model has long-term benefit on the behavioral outcome [14]. Specific growth factors utilized in this preclinical study were: brain-derived neurotrophic factor, epidermal growth factor, human chorionic gonadotropin, and erythropoietin (Epo) $[14,17,18]$. However, similar to the sparsity of human trials in examining the inflammatory process of a stroke, human trials are also spare when discussing neuronal regeneration via administering growth factors following a stroke.

Preclinical studies that examined the efficacy of Epo in promoting neural repair suggests that Epo is beneficial when administered as a monotherapy within $24-48$ hours after the onset of the ischemic stroke. Epo was discovered to be not only beneficial but also safe for patients. In a randomized, placebo-controlled study, 167 patients received two doses of Epo and no adverse side effects were reported when comparing epo versus placebo $[19,20]$. However, high dose Epo administered along with tPA had an increased mortality rate in comparison to the placebo group. As a result of the fatal interaction between Epo and tPA, further studies are needed to determine if the interaction is dose-dependent or if Epo can never be administered in conjunction with tPA.

The majority of human trials regarding neuronal regeneration as a result of growth factors examine hematopoetic growth factors in relation to ischemic strokes. In particular, granulocytecolony stimulating factor (G-CSF) was evaluated in the AX200 for Ischemic Stroke Study (AXIS). The AXIS study concluded that G-CSF is tolerated when given within 12 hours of an ischemic stroke, however, a meta-analysis conducted after the AXIS study discovered that administration of G-CSF at any time span, days to years, post-stroke had unfavorable effects [2124]. 


\subsection{Monoclonal Antibodies}

Along with growth factors, MAB can also be used to promote axonal growth. Specifically, there are three major inhibitors of neuronal regeneration that are targets of such MABs: myelinassociated glycoprotein (MAG), oligo-myelin glycoprotein, and Nogo-A. Following an ischemic stroke, these inhibitors of neuronal regeneration are upregulated and decrease neuronal growth and regeneration $[8,25]$.

To examine the importance of neutralizing the inhibitors of neuronal growth, one study randomized 42 patients into a placebo group or the group receiving GSK249320. GSK249340 is an IgG1 MAB with a disabled Fc region directed against MAG. To measure an increase in neuronal regeneration, gait velocity was evaluated. Initially, gait velocity showed greater improvement in those that received GSK249320 in comparison to placebo. However, in a phase IIb double-blind study, it was discovered that two doses of GSK249320 did not improve gait velocity in comparison to placebo $[26,27]$. Anti-MAG MAB was also shown to reduce the volume of the lesion when administered at $0.05 \mu \mathrm{g} / \mathrm{mL} 1$ hour after MCAO in animal models. Animals treated with anti-MAG antibody showed significant improvements in total neurological score at 24 hours, 48 hours, and 7 days after treatment when compared to those of the placebo group. Stroke lesions were reduced by $40-50 \%$ compared to the placebo [28].

Nogo-A is produced by oligodendrocytes and is another contributing factor to the inhibition of neuronal growth after CNS injury. However, the best time to inhibit Nogo-A following an ischemic stroke has not been identified. Nogo-A cannot be inhibited immediately following an ischemic stroke. It has been proven that Nogo-A is initially involved in healing after a stroke but eventually becomes an inhibitor, yet the timespan of such events is uncertain [11]. Mice that were depleted of Nogo-A had increased mortality after stroke in an MCAO 
model. Likewise, mice that were administered anti-Nogo-A immediately following MCAO had a similar increase in mortality as knock-out mice. Therefore, Nogo-A must play a necessary role in healing immediately following a stroke. However, immunotherapy against Nogo-A that was administered 7-9 days after an ischemic stroke proved beneficial. The administration of antiNogo-A a week after MCAO in mice showed increased sprouting, increased neuroplasticity, increased midline crossing of corticorubral axons to the red nucleus of the cerebellum, and new efferent cortical projections [11]. Another study evaluated the importance of antibodies directed against Nogo-A and discovered that forepaw function drastically improved [29]. Purified monoclonal anti-Nogo-A antibody (7B12) was administered to rats exactly 24 hours after MCAO induction. Forepaw function of rats that had MCAO without 7B12 improved to 40-50\% of prelesion levels from weeks 4 to 12 after MCAO. In contrast, the 7B12 group had improved to $40 \%$ of prelesion levels at week 4 with $60-70 \%$ when evaluated at weeks 7-12 [29].

\subsection{MAB acts on Acid-Sensing Ion Channels}

Acid-sensing ion channels (ASICs) are another therapeutic target for ischemic stroke. ASICs are voltage-insensitive cation channels present on cell membranes that are responsible for detecting the acidic microenvironment of cells [30-32]. ASICs are activated in an acidic microenvironment $[33,34]$. During an ischemic stroke, oxygen and glucose deprivation results in anaerobic metabolism. Anaerobic metabolism leads to an accumulation of lactic acids, and this decrease in $\mathrm{pH}$ activates neuronal ASICs $[34,35]$. The activation of ASICs leads to an influx of calcium which contributes to neuronal cell death [35]. Specifically, ASIC1a is calcium permeable and is a therapeutic target for ischemic strokes [36 - 38]. 
A MAB called ASC06- IgG1 has been discovered that specifically blocks ASIC1a [36]. An MCAO stroke was induced in mice, and three hours after ischemia, $4 \mu \mathrm{L}$ of PBS (vehicle solution) $+3 \mu \mathrm{g} / \mu \mathrm{L}$ ASC06- IgG1 was injected intracerebroventricularly. Compared to the control, the group treated with PBS + ASC06-IgG1 showed a 23\% decrease in infarct volume [36]. Such results show that ASICs contribute to neuronal cell death. Antibodies against ASICs are neuroprotective and might have therapeutic potential in stroke patient [36].

\subsection{MAB acts on $N$-methyl-D-aspartate (NMDA) receptors}

$N$-methyl-D-aspartate receptors (NMDARs) are activated during an ischemic stroke.

During an ischemic stroke, glutamate is released into the plasma and cerebrospinal fluid from damaged neuronal tissue; an increase in glutamate leads to activation of NMDARs [39]. Once glutamate binds to NMDARs, there is a subsequent influx in calcium. The overload of intracellular calcium contributes to neuronal cell death during an ischemic stroke [40-43].

An anti-GluN1 antibody was developed in animal models. Anti-GluN1 antibodies were shown to decrease the infarct volume after an ischemic stroke was induced in rats by interfering with activated neuronal NMDARs. The vaccine was able to cross into the brain due to the destruction of the blood brain barrier following an ischemic stroke [44].

It is hypothesized that anti-GluN1 antibodies provide therapeutic benefits following an ischemic stroke by inhibiting platelet function [39]. Rats were vaccinated with GluN1 and were evaluated based on the antibodies formed. Various rat anti-GluN1 antibodies were cloned and evaluated based on human platelet aggregation. The study has shown that rats vaccinated with GluN1 have prolonged bleeding and anti-GluN1-S2 antibodies inhibit human platelet 
aggregation and reduce growth of thrombi [39]. However, anti-GluN1-S2 antibodies have not been directly tested in stroke models [39].

\section{Conclusion}

While the current clinical treatment for patients suffered from ischemic strokes is tPA, the narrow therapeutic window of less than 4.5-hours from the onset of symptoms limits its benefit as a treatment $[2,3]$. However, recent studies have examined emerging therapies to treat beyond the narrow timeframe of tPA such as activating growth factors, inhibiting proinflammatory cytokines, enhancing anti-inflammatory cytokines, and blocking ion channels through MABs. By increasing the time window allowed for treatment from hours as provided by tPA to days as allowed with such experimental therapies, a larger patient population will benefit from treatment and the lifelong side-effects of an ischemic stroke can decrease [3]. Therefore, developing specific MAB targeting the signaling pathway of stroke will reveal a great potential in stroke therapy.

Author Contributions: D.W., Q.J., X.-P.C. wrote the manuscript.

Funding: Demi Woods received Sarah Morrison Research Award from the University of Missouri-Kansas City School Medicine, Missouri, USA. Xiang-Ping Chu received the research support from University of Missouri Research Board, Missouri, USA, the Qiqihar Medical University (QY2016-ZD1), Heilongjiang Province, China and American Heart Association (19AIREA34470007).

Conflicts of Interest: D.W., Q.J., X.-P.C. declare no conflict of interest. 


$\begin{array}{ll}\text { Abbreviations } & \\ \text { ASICs } & \text { acid-sensing ion channels } \\ \text { AXIS } & \text { AX200 for Ischemic Stroke Study } \\ \text { CNS } & \text { central nervous system } \\ \text { Epo } & \text { erythropoietin } \\ \text { FDA } & \text { Federal Drug and Food Administration } \\ \text { G-CSF } & \text { granulocyte-colony stimulating factor } \\ \text { IL } & \text { interleukin } \\ \text { IP-10 } & \text { interferon-inducible protein 10 } \\ \text { MAB } & \text { monoclonal antibody } \\ \text { MAG } & \text { myelin-associated glycoprotein } \\ \text { MCAO } & \text { middle cerebral artery occlusion } \\ \text { MCP-1 } & \text { monocyte chemoattraction protein-1 } \\ N M D A & N \text {-methyl-D-aspartate } \\ N M D A R s & N \text {-methyl-D-aspartate receptors } \\ \text { TGF } & \text { transforming growth factor } \\ \text { TLR4 } & \text { toll-like receptor 4 } \\ \text { TNF } & \text { tumor necrosis factor } \\ \text { tPA } & \text { tissue plasminogen activator }\end{array}$

\section{References}

1. Zhou, Z.; Lu, J.; Liu, W.W.; Manaenko, A.; Hou, X.; Mei, Q.; Huang, J.L.; Tang, J.; Zhang, J.H.; Yao, H.; Hu, Q. Pharmacol. Ther. 2018, 191, 23-42.

2. Siniscalchi, A.; Iannacchero, R.; Anticoli, S.; Romana Pezzella, F.; De Sarro, G.; Gallelli, L. Anti-inflammatory strategies in stroke: a potential therapeutic target. Curr. Vasc. Pharmacol. 2016, 14(1), 98-105.

3. National Institute of Neurological Disorders and Stroke rt-PA Stroke Study Group. Tissue plasminogen activator for acute ischemic stroke. N. Engl. J. Med. 1995, 333, 1581-1587.

4. Yu, C.Y.; Ng, G.; Liao, P. Therapeutic antibodies in stroke. Transl. Stroke Res. 2013, $4(5), 477-483$.

5. Posada-Duque, R.A.; Barreto, G.E.; Cardona-Gomez, .GP. Protection after stroke: cellular effectors of neurovascular unit integrity. Front. Cell. Neurosci. 2014, 8, 231. 
6. Deftereos, S.; Angelidis, C.; Bouras, G.; Raisakis, K.; Gerckens, U.; Cleman, M.W.; Giannopoulos, G. Innate immune inflammatory response in the acutely ischemic myocardium. Med. Chem. 2014, 10(7), 653-662.

7. Banwell, V.; Sena, E.S.; Macleod, M. R. Systematic review and stratified meta-analysis of the efficacy of interleukin-1 receptor antagonist in animal models of stroke. J. Stroke Cerebrovasc. Dis. 2009, 18(4), 269-276.

8. Li, S.; Carmichael, S.T. Growth-associated gene and protein expression in the region of axonal sprouting in the aged brain after stroke. Neurobiol. Dis. 2006, 23, 362-373.

9. Cheatwood, J.L.; Emerick, A.J.; Schwab, M.E.; Kartje, G.L. Nogo-A expression after focal ischemic stroke in the adult rat. Stroke 2008, 39, 2091-2098.

10. Nilupul Perera, M.; Ma, H.K.; Arakawa, S.; Howells, D.W.; Markus, R.; Rowe, C.C.; Donnan, G.A. Inflammation following stroke. J. Clin. Neurosci. 2006, 13, 1- 8.

11. Yu, C.Y.; Ng, G.; Liao, P. Therapeutic antibodies in stroke. Transl. Stroke Res. 2013, $4(5), 477-483$.

12. Andresen, L.; Theodorou, K.; Grünewald, S.; Czech-Zechmeister, B.; Könnecke, B.; Lühder, F.; Trendelenburg, G. Evaluation of the Therapeutic Potential of Anti-TLR4Antibody MTS510 in Experimental Stroke and significance of different routes of application. PloS. One 2016, 11(2), e0148428.

13. Elkins, J.; Veltkamp, R.; Montaner, J.; Johnston, S.C.; Singhal, A.B.; Becker, K.; Lansberg, M.G.; Tang, W.; Chang, I.; Muralidharan, K.; Gheuens, S.; Meht,a L.; Elkind, M.S.V. Safety and efficacy of natalizumab in patients with acute ischaemic stroke (ACTION): a randomised, placebo-controlled, double-blind phase 2 trial. Lancet Neurol. 2017, 16(3), 217-226.

14. Belayev, L.; Khoutorova, L.; Zhao, K.L.; Davidoff, A.W.; Moore, A.F.; Cramer, S.C. A novel neurotrophic therapeutic strategy for experimental stroke. Brain Res. 2009, 1280, 117-123.

15. Lanfranconi, S.; Locatelli, F.; Corti, S.; Candelise, L.; Comi, G.P.; Baron, P.L.; Strazzer, S.; Bresolin, N.; Bersano, A. Growth factors in ischemic stroke. J. Cell. Mol. Med. 2011, $15,1645-1687$.

16. Oliveira, S.L.; Pillat, M.M.; Cheffer, A.; Lameu, C.; Schwindt, T.T.; Ulrich, H. Functions of neurotrophins and growth factors in neurogenesis and brain repair. Cytometry A 2013, $83,76-89$.

17. Ren, J.M.; Finklestein, S.P. Growth factor treatment of stroke. Curr. Drug Targets CNS Neurol. Disord. 2005, 4, 121-125. 
18. Kolb, B.; Morshead, C.; Gonzalez, C.; Kim, M.; Gregg, C.; Shingo, T.; Weiss, S. Growth factor-stimulated generation of new cortical tissue and functional recovery after stroke damage to the motor cortex of rats. J. Cereb. Blood Flow Metab. 2007, 27, 983-997.

19. Jerndal, M.; Forsberg, K.; Sena, E.S.; Macleod, M.R.; O’Collins, V.E.; Linden, T.; Nilsson, M.; Howells, D.W. A systematic review and meta-analysis of erythropoietin in experimental stroke. J. Cereb. Blood Flow Metab. 2010, 30, 961-968.

20. Yip, H.K.; Tsai, T.H.; Lin, H.S.; Chen, S.F.; Sun, C.K.; Leu, S.; Yuen, C.M.; Tan, T.Y.; Lan, M.Y.; Liou, C.W.; Lu, C.H.; Chang, W.N. Effect of erythropoietin on level of circulating endothelial progenitor cells and outcome in patients after acute ischemic stroke. Crit. Care 2011, 15, R40.

21. Schäbitz, W.R.; Laage, R.; Vogt, G.; Koch, W.; Kollmar, R.; Schwab, S.; Schneider, D.; Hamann, G.F.; Rosenkranz, M.; Veltkamp, R.; Fiebach, J.B.; Hacke, W.; Grotta, J.C.; Fisher, M.; Schneider, A. AXIS: a trial of intravenous granulocyte colony stimulating factor in acute ischemic stroke. Stroke 2010, 41, 2545-2551.

22. England, T.J.; Abaei, M.; Auer, D.P.; Lowe, J.; Jones, D.R.; Sare, G.; Walker, M.; Bath, P.M. Granulocyte-colony stimulating factor for mobilizing bone marrow stem cells in subacute stroke: the stem cell trial of recovery enhancement after stroke 2 randomized controlled trial. Stroke 2012, 43, 405-411.

23. Ringelstein, E.B.; Thijs, V.; Norrving, B.; Chamorro, A.; Aichner, F.; Grond, M.; Saver, J.; Laage, R.; Schneider, A.; Rathgeb, F.; Vogt, G.; Charissé, G.; Fiebach, J.B.; Schwab, S.; Schäbitz, W.R.; Kollmar, R.; Fisher, M.; Brozman, M.; Skoloudik, D.; Gruber, F.; Serena Leal, J.; Veltkamp, R.; Köhrmann, M.; Berrouschot, J.; AXIS 2 Investigators. Granulocyte colony-stimulating factor in patients with acute ischemic stroke: results of the AX200 for Ischemic Stroke trial. Stroke 2013, 44, 2681-2687.

24. England, T.J.; Sprigg, N.; Alasheev, A.M.; Belkin, A.A.; Kumar, A.; Prasad, K.; Bath, P.M. Granulocyte-colony stimulating factor (G-CSF) for stroke: an individual patient data meta-analysis. Sci. Rep. 2016, 6, 36567.

25. Cheatwood, J.L.; Emerick, A.J.; Schwab, M.E.; Kartje, G.L. Nogo-A expression after focal ischemic stroke in the adult rat. Stroke 2008, 39, 2091-2098.

26. Domeniconi, M., Filbin, M.T. Overcoming inhibitors in myelin to promote axonal regeneration. J. Neurol. Sci. 2005, 233, 43-47.

27. Buchli, A.D., Schwab, M.E. Inhibition of Nogo: a key strategy to increase regeneration, plasticity and functional recovery of the lesioned central nervous system. Ann. Med. 2005, 37, 556-567.

28. Irving, E.A.; Vinson, M.; Rosin, C.; Roberts, J.C.; Chapman, D.M.; Facci, L.; Virley, D.J.; Skaper, S.D.; Burbidge, S.A.; Walsh, F.S.; Hunter, A.J.; Parsons, A.A. 
Identification of Neuroprotective Properties of Anti-MAG Antibody: A Novel Approach for the Treatment of Stroke? J. Cereb. Blood Flow Metab. 2005, 25(1), 98-107.

29. Wiessner, C.; Bareyre, F.M.; Allegrini, P.R.; Mir, A.K.; Frentzel, S.; Zurini, M.; Schnell, L.; Oertle, T.; Schwab, M.E. Anti-Nogo-A Antibody Infusion 24 Hours after Experimental Stroke Improved Behavioral Outcome and Corticospinal Plasticity in Normotensive and Spontaneously Hypertensive Rats. J. Cereb. Blood Flow Metab. 2003, 23(2), 154-165.

30. Waldmann, R.; Champigny, G.; Bassilana, F.; Heurteaux, C.; Lazdunski, M. A protongated cation channel involved in acid-sensing. Nature 1997, 386, 173-177.

31. Kellenberger, S.; Schild, L. International Union of Basic and Clinical Pharmacology. XCI. structure, function, and pharmacology. Pharmacol Rev 2015, 67(1), 1-35.

32. Wemmie, J.A.; Taugher, R.J.; Kreple, C.J. Acid-sensing ion channels in pain and disease. Nat. Rev. Neurosci. 2013, 14, 461-471.

33. Rash, L.D. Acid-sensing ion channel pharmacology, past, present, and future. $A d v$. Pharmacol. 2017, 79, 35-66.

34. Chu, X.P.; Grasing, K.A.; Wang, J.Q. Acid-sensing ion channels contribute to neurotoxicity. Transl. Stroke Res. 2014, 5(1), 69-78.

35. Xiong, Z.G.; Zhu, X.M.; Chu, X.P.; Minami, M.; Hey, J.; Wei, W.L.; MacDonald, J.F.; Wemmie, J.A.; Price, M.P.; Welsh, M.J.; Simon, R.P. Neuroprotection in ischemia: blocking calcium-permeable acid-sensing ion channels. Cell 2004, 118(6), 687-698.

36. Qiang, M.; Dong, X.; Zha, Z.; Zuo, X.K.; Song, X.L.; Zhao, L.; Yuan, C.; Huang, C.; Tao, P.; Hu, Q.; Li, W.G.; Hu, W.; Li, J.; Nie, Y.; Buratto, D.; Zonta, F.; Ma, P.; Yu, Z.; Liu, L.; Zhang, Y.; Yang, B.; Xie, J.; Xu, T.L.; Qu, Z.; Yang, G.; Lerner, R.A. Selection of an ASIC1a- blocking combinatorial antibody that protects cells from ischemic death. Proc. Natl. Acad. Sci. U S A. 2018, 115(32), E7469-E7477.

37. Baron, A.; Lingueglia, E. Pharmacology of acid-sensing ion channels-Physiological and therapeutical perspectives. Neuropharmacology 2015, 94, 19-35.

38. Li, M.H.; Inoue, K.; Si, H.F.; Xiong, Z.G. Calcium-permeable ion channels involved in glutamate receptor-independent ischemic brain injury. Acta Pharmacol. Sin. 2011, 32, 734-740.

39. Green, T.N.; Hamilton, J.R.; Morel-Kopp, M.C.; Zheng, Z.; Chen, T.T.; Hearn, J.I.; Sun, P.P.; Flanagan, J.U.; Young, D.; Barber, P.A.; During, M.J.; Ward, C.M.; KalevZylinska, M.L. Inhibition of NMDA receptor function with an anti-GluN1-S2 antibody impairs human platelet function and thrombosis. Platelets 2017, 28(8), 799-811. 
40. Traynelis, S.F.; Wollmuth, L.P.; McBain, C.J.; Menniti, F.S.; Vance, K.M.; Ogden, K.K.; Hansen, K.B.; Yuan, H.; Myers, S.J.; Dingledine, R. Glutamate receptor ion channels: structure, regulation, and function. Pharmacol. Rev. 2010, 62,405-496.

41. Paoletti, P.; Bellone, C.; Zhou, Q. NMDA receptor subunit diversity: impact on receptor properties, synaptic plasticity and disease. Nat. Rev. Neurosci. 2013, 14,383-400.

42. Szydlowska, K.; Tymianski, M. Calcium, ischemia and excitotoxicity. Cell Calcium 2010, 47,122-129.

43. Forder, J.P.; Tymianski, M. Postsynaptic mechanisms of excitotoxicity: Involvement of postsynaptic density proteins, radicals, and oxidant molecules. Neuroscience 2009, 158, 293-300.

44. During, M.J.; Symes, C.W.; Lawlor, P.A.; Lin, J.; Dunning, J.; Fitzsimons, H.L.; Poulsen, D.; Leone, P.; Xu, R.; Dicker, B.L.; Lipski, J.; Young, D. An oral vaccine against NMDAR1 with efficacy in experimental stroke and epilepsy. Science 2000, 287, $1453-1460$. 